\title{
Screening of Lactobacillus spp. for the prevention of Pseudomonas aeruginosa pulmonary infections
}

\author{
Youenn Alexandre ${ }^{1}$, Rozenn Le Berre ${ }^{1,2^{*}}$, Georges Barbier ${ }^{3}$ and Gwenaelle Le Blay ${ }^{3,4}$
}

\begin{abstract}
Background: Pseudomonas aeruginosa is an opportunistic pathogen that significantly increases morbidity and mortality in nosocomial infections and cystic fibrosis patients. Its pathogenicity especially relies on the production of virulence factors or resistances to many antibiotics. Since multiplication of antibiotic resistance can lead to therapeutic impasses, it becomes necessary to develop new tools for fighting $P$. aeruginosa infections. The use of probiotics is one of the ways currently being explored. Probiotics are microorganisms that exert a positive effect on the host's health and some of them are known to possess antibacterial activities. Since most of their effects have been shown in the digestive tract, experimental data compatible with the respiratory environment are strongly needed. The main goal of this study was then to test the capacity of lactobacilli to inhibit major virulence factors (elastolytic activity and biofilm formation) associated with $P$. aeruginosa pathogenicity.

Results: Sixty-seven lactobacilli were isolated from the oral cavities of healthy volunteers. These isolates together with 20 lactobacilli isolated from raw milks, were tested for their capacity to decrease biofilm formation and activity of the elastase produced by $P$. aeruginosa PAO1. Ten isolates, particularly efficient, were accurately identified using a polyphasic approach (API $50 \mathrm{CHL}$, mass-spectrometry and 16S/rpoA/phes genes sequencing) and typed by pulsed-field gel electrophoresis (PFGE). The 8 remaining strains belonging to the L. fermentum (6), L. zeae (1) and L. paracasei (1) species were sensitive to all antibiotics tested with the exception of the intrinsic resistance to vancomycin. The strains were all able to grow in artificial saliva.

Conclusion: Eight strains belonging to L. fermentum, L. zeae and L. paracasei species harbouring anti-elastase and anti-biofilm properties are potential probiotics for fighting P. aeruginosa pulmonary infections. However, further studies are needed in order to test their innocuity and their capacity to behave such as an oropharyngeal barrier against Pseudomonas aeruginosa colonisation in vivo.
\end{abstract}

Keywords: Pseudomonas aeruginosa, Lactobacillus, Probiotics, Organic acids, Biofilm formation, Elastolytic activity

\section{Background}

Pseudomonas aeruginosa is one of the most common pathogens responsible for acute respiratory infections in ventilated or immunocompromised patients, and for chronic respiratory infections in cystic fibrosis (CF) patients. Between 1975 and 2003, the frequency of hospitalacquired pneumonia caused by $P$. aeruginosa increased from $9.6 \%$ to $18.1 \%$, this pathogen thus becoming the main cause of acute respiratory infections [1]. Mortality

\footnotetext{
* Correspondence: rozenn.leberre@chu-brest.fr

${ }^{1}$ Université de Brest, EA 3882-Laboratoire Universitaire de Biodiversité et d'Écologie Microbienne (LUBEM), Faculté de Médecine, 22 avenue Camille Desmoulins, 29200 Brest, France

2Département de Médecine Interne et Pneumologie, CHRU La

Cavale-Blanche, 29200 Brest, France

Full list of author information is available at the end of the article
}

rates in ventilator-associated pneumonia caused by $P$. aeruginosa range from 42.1 to $87 \%$ [2]. This bacteria is also associated with over $80 \%$ of the morbidity and mortality rates in CF patients [3]. These features may be explained by the wide range of both cellular associated and extracellular virulence factors involved in the pathogenesis of $P$. aeruginosa pneumonia $[4,5]$. Indeed, $P$. aeruginosa is endowed with remarkable virulence factors like lipopolysaccharide, type III secretion system, pyocyanin and elastase. Moreover, it is intrinsically resistant to a large number of antibiotics and can acquire resistances to many others. $P$. aeruginosa may also form biofilms that protect it from the host immune system, while decreasing antibiotics accessibility and increasing the difficulties of eradication in CF patients particularly [6]. Therapies based on the 
exclusive use of antibiotics may then lead to therapeutic impasses and it is necessary to find new therapeutic options to fight $P$. aeruginosa pulmonary infections.

In this context, the use of probiotic bacteria, either as prophylactic agents for preventing or delaying pulmonary colonisation with $P$. aeruginosa, or eventually as therapeutic tool to fight $P$. aeruginosa infections, seems to be particularly attractive.

The emergence of the microbiota concept, with the accumulation of evidences that human associated microbiota play a major role in health and disease $[7,8]$, induced a profound modification in the perception of probiotics. For long confined to the gastrointestinal tract, where most of their positive effects have been described [9], they are now covering a much broader domain of applications. Evidence is emerging that probiotics may have a primordial role in health of the oral cavity [10] or in preventing mechanically ventilated patients from ventilator associated pneumonia. Several randomized controlled trials directly exploring the role of probiotics in preventing ventilator-associated pneumonia were published $[11,12]$. Even if the conclusions are controversial, probably because of the heterogeneity of the used probiotic strains, of the mode of administration, of the clinical situations and of the primary endpoint, this strategy seems promising. The lack of rational work for the selection of probiotic strains adapted to the respiratory ecosystem may be also one of the reasons explaining the poor results obtained in certain studies.

In this context, the main objective of this work was the screening for putative probiotic strains active against $P$. aeruginosa. Eighty-seven lactobacilli isolates, isolated from the oral cavity or raw milk, were tested for their capacity to inhibit elastolytic activity and biofilm formation, two main virulence factors of $P$. aeruginosa. In a second step, the most active isolates were characterized with molecular and phenotypic methods, and their antibiotic resistance and growth capacity in artificial saliva were checked.

\section{Results}

\section{Isolation and identification of oral lactobacilli}

Sixty-seven colonies were isolated from the oral cavities of 23 healthy volunteers. Among them, 7 isolates were assigned at the genus level only (Lactobacillus) by MALDI-TOF spectrometry analyses with scores below 1.8, whereas the majority (60 isolates) were assigned at the species level with scores above 1.8. They belonged to 9 Lactobacillus species (Table 1), with a strong representativeness of the Lactobacillus reuteri and Lactobacillus casei groups with respectively 40 (with a predominance of L. fermentum) and 21 isolates (with a predominance of $L$. paracase $i$ ). The isolation and characterization strategies of bacterial isolates are shown
Table 1 Preliminary identification (MALDI-TOF analyses) of newly isolated oral lactobacilli

\begin{tabular}{ccc}
\hline Lactobacillus groups* $^{*}$ & Species** $^{*}$ & Isolates \\
\hline L. reuteri & L. reuteri & 1 \\
& L. fermentum & 30 \\
L. vaginalis & 3 \\
L. casei & L. zeae/casei & 5 \\
& L. rhamnosus & 6 \\
L. salivarius & L. paracasei & 10 \\
L. plantarum & L. salivarius & 4 \\
\hline
\end{tabular}

*According to Felis and Dellaglio [14], **MALDI-ToF scores above 1.8 .

in Figure 1. These 67 isolates were pooled with 20 lactobacilli from a collection of bacteria isolated from raw milks [13].

\section{Effects of $\mathrm{pH}$ and acids on $P$. aeruginosa growth}

$P$. aeruginosa strains are known to be sensitive to organic acids [15,16]. Since Lactobacillus spp. produce lactic and acetic acids that inhibit the growth of many bacteria through their undissociated forms at low $\mathrm{pH}$, the sensitivity of $P$. aeruginosa PAO1 was tested toward both of them at different $\mathrm{pH}$. P. aeruginosa PAO1 growth was monitored in LB broth by measuring $\mathrm{OD}_{595 \mathrm{~nm}}$ values at four $\mathrm{pH}(7,6,5$ or 4$)$, in presence $(50$ or $100 \mathrm{mM})$ or absence of lactic acid. These results are presented in Table 2. P. aeruginosa was sensitive to the $\mathrm{pH}$ decrease with a pronounced growth inhibition at $\mathrm{pH} 5$ and 4 $(p<0.0001)$. However, no specific effect of lactic acid was observed $(p=0.21)$. The addition of acetic acid induced different effects (Table 2). As observed previously, the $\mathrm{pH}$ induced a significant decrease of $P$. aeruginosa $(p<0.0001)$, but in that case the addition of acetic acid induced a significant reduction of $P$. aeruginosa growth at $\mathrm{pH} 6$ (from $50 \mathrm{mM}$ of acetic acid and higher) and at $\mathrm{pH} 5$ (from $25 \mathrm{mM}$ of acetic acid and higher). No specific effect of acetic acid was detected on $P$. aeruginosa at $\mathrm{pH} 4$ (no growth) nor at $\mathrm{pH} 7$ (growth), whatever its concentration.

\section{Effects of lactobacilli isolates on biofilm formation}

Eighty-seven Lactobacillus isolates (67 from the oral cavity and 20 from raw milk) were tested for their capacity to inhibit biofilm formation by $P$. aeruginosa PAO1 (Table 3). As compared with the positive control ( $P$. aeruginosa PAO1 alone), only five isolates from the oral cavity significantly $(p<0.05)$ reduced the amount of biofilm formation after $7 \mathrm{~h}$ of co-incubation with $P$. aeruginosa PAO1 at $37^{\circ} \mathrm{C}$ in BHI broth (Table 3). The four L. fermentum isolates ES.A.2, ES.F.115, ES.A.1a and ES.A.6a induced a biofilm reduction of $3,7,10$ and $11 \%$ respectively, whereas $L$. paracasei ES.D.88 induced a reduction of 


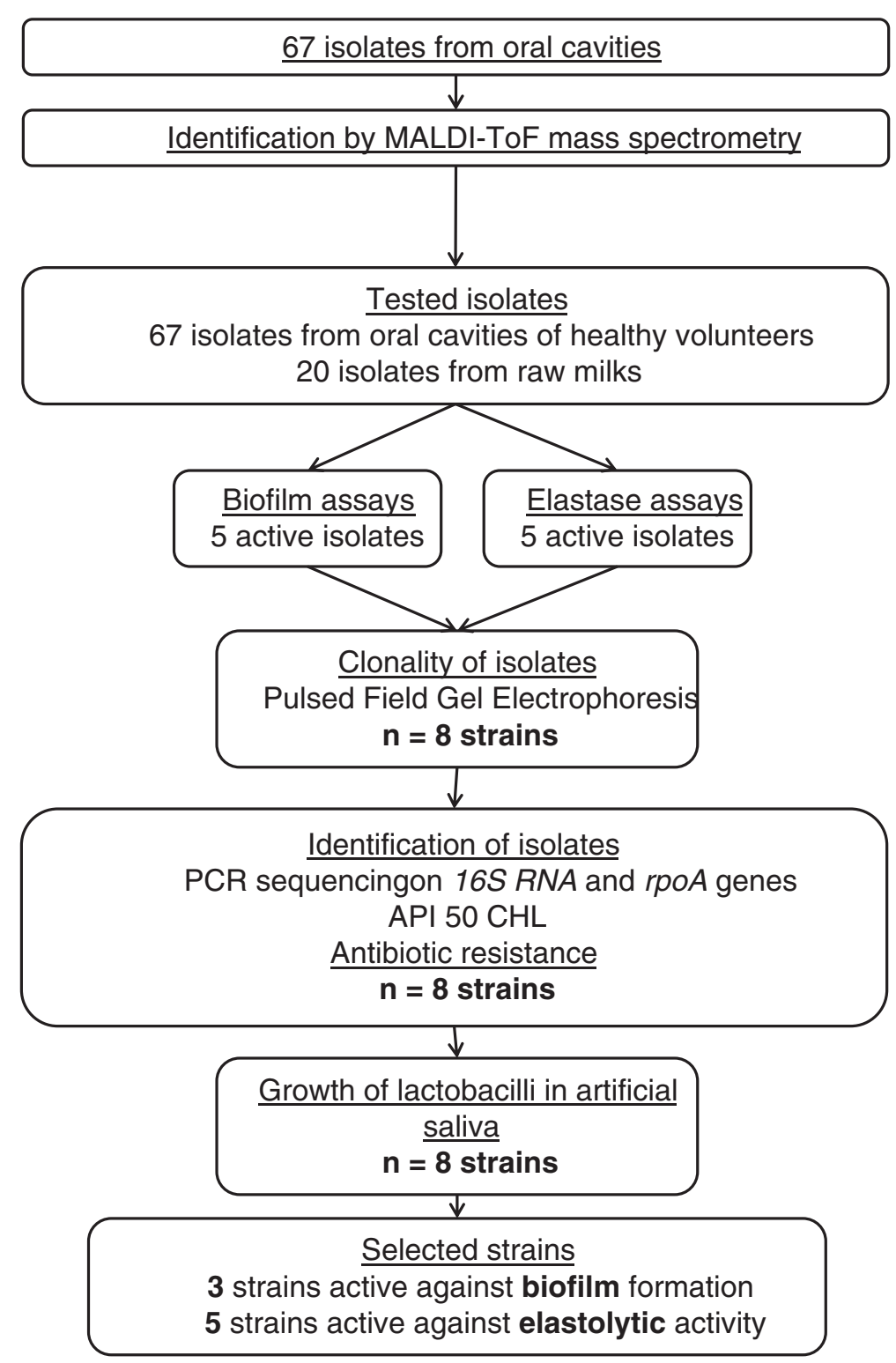

Figure 1 Flow-chart of the strains selection strategy.

15\%. Despite a $\mathrm{pH}$ decrease of the BHI medium ( $\mathrm{pH} 7.4)$ ranging from 0.6 to 1 unit during the $7 \mathrm{~h}$ of co-incubation, no growth inhibition of $P$. aeruginosa was observed.

\section{Effects of lactobacilli isolates on elastolytic activity}

The same eighty-seven isolates were tested for their capacity to inhibit the elastolytic activity of $P$. aeruginosa PAO1. Only five of them significantly $(p<0.001)$ reduced the activity of elastase after $22 \mathrm{~h}$ of co-incubation with $P$. aeruginosa $\mathrm{PAO} 1$ at $37^{\circ} \mathrm{C}$ in $\mathrm{BHI}$ broth, as compared with the positive control ( $P$. aeruginosa PAO1 alone) (Table 3). Among them, 4 strains (K.C6.3.1D, K.V9.3.2B, K.V9.3.2C and K.C6.3.1E) were L. fermentum isolated from raw milk. They respectively reduced by $37 \%$, 38\%,
$38 \%$ and $47 \%$ the elastolytic activity of $P$. aeruginosa. Only one strain isolated from the oral cavity ( $L$. zeae Od.76), significantly reduced (minus 36\%) the elastolytic activity. When grown in presence of lactobacilli during $22 \mathrm{~h}$ and despite a $\mathrm{pH}$ decrease ranging from 0.9 to 1.2 units of the BHI medium ( $\mathrm{pH} 7.4$ ), no inhibition of $P$. aeruginosa growth was observed.

\section{Formal identification of lactobacilli active against P. aeruginosa}

Once several isolates were shown to be active against $P$. aeruginosa PAO1, their identity was confirmed by $16 S$ $r R N A$ and $r p o A$ genes sequencing, and API $50 \mathrm{CHL}$ (Table 4, Additional file 1). Two isolates (ES.D.88 and 
Table 2 In vitro effects of $\mathrm{pH}$, lactic and acetic acids on Pseudomonas aeruginosa PAO1 growth

\begin{tabular}{|c|c|c|c|c|}
\hline Acid concentrations (mM) & & aeruginosc & and acid co & \\
\hline Lactic acid & $\mathrm{pH} 7$ & $\mathrm{pH} 6$ & $\mathrm{pH} 5$ & $\mathrm{pH} 4$ \\
\hline 0 & $0.60 \pm 0.03$ & $0.62 \pm 0.01$ & $0.43 \pm 0.02$ & $0.01 \pm 0.00$ \\
\hline 50 & $0.74 \pm 0.06$ & $0.81 \pm 0.11$ & $0.47 \pm 0.19$ & $0.12 \pm 0.01$ \\
\hline 100 & $0.65 \pm 0.02$ & $0.65 \pm 0.02$ & $0.23 \pm 0.07$ & $0.12 \pm 0.01$ \\
\hline Acetic acid & $\mathrm{pH} 7$ & $\mathrm{pH} 6$ & $\mathrm{pH} 5$ & $\mathrm{pH} 4$ \\
\hline 0 & $0.60 \pm 0.03$ & $0.62 \pm 0.01$ & $0.43 \pm 0.02$ & $0.01 \pm 0.00$ \\
\hline 12.5 & $0.70 \pm 0.06$ & $0.58 \pm 0.02$ & $0.38 \pm 0.27$ & $0.12 \pm 0.01$ \\
\hline 25 & $0.63 \pm 0.06$ & $0.65 \pm 0.09$ & $0.11 \pm 0.01$ & $0.12 \pm 0.00$ \\
\hline 50 & $0.56 \pm 0.02$ & $0.26 \pm 0.07$ & $0.11 \pm 0.01$ & $0.12 \pm 0.01$ \\
\hline 100 & $0.77 \pm 0.14$ & $0.10 \pm 0.01$ & $0.11 \pm 0.01$ & $0.13 \pm 0.01$ \\
\hline
\end{tabular}

*Each value is the mean of triplicates \pm SD (ANCOVA), $P$. aeruginosa was incubated in BHI medium for $22 \mathrm{~h}$ in presence of different acid concentrations (acetic, lactic acid or $\mathrm{HCl}$ ).

Od. 76) were identified as L. paracasei and L. zeae respectively. In the last case, it was not possible to formally distinguish between the two closely related $L$. zeae and $L$. casei species by sequencing $16 S$ RNA and rpo $A$ genes only. The sequencing of pheS gene (Genbank accession number: KJ402364) was then necessary to formally assigned the Od.76 strain to the L. zeae species. The eight other active isolates (ES.A.1a, ES.A.2, ES.A.6a, ES.F.115, K. C6.3.1D, K.C6.3.1E, K.V9.3.2B and K.V9.3.2C) were shown to belong to L. fermentum. A pulsed-field gel electrophoresis (PFGE) showed that ES.A.1a, ES.A.2 and ES.A.6a were clonal strains (data not shown). ES.A.2 was then the only one kept for the last tests. The API $50 \mathrm{CHL}$ gallery confirmed these results for all strains but one, L. zeae Od.76, since this method is not designed to identify L. casei nor L. zeae.

\section{Antibiotics sensitivity of lactobacilli}

All the strains were resistant to vancomycin, as expected for lactobacilli. No other resistance was detected against the 9 other antibiotics for any of the tested strains (ampicillin, vancomycin, gentamicin, kanamycin, streptomycin,

Table 3 In vitro relative effects of lactobacilli isolates against biofilm formation and elastolytic activity of Pseudomonas aeruginosa PAO1

\begin{tabular}{lccc}
\hline \multicolumn{2}{c}{ Biofilm formation* } & \multicolumn{2}{c}{ Elastolytic activity $^{*}$} \\
\hline \multicolumn{1}{c}{ Control } & $100 \%$ & Control & $100 \%$ \\
L. fermentum ES.A.2 & $95 \%$ & L. fermentum K.C6.3.1D & $63 \%$ \\
L. fermentum ES.F.115 & $93 \%$ & L. zeae Od.76 & $64 \%$ \\
L. fermentum ES.A.6a & $88 \%$ & L. fermentum K.V9.3.2B & $62 \%$ \\
L. fermentum ES.A.1a & $88 \%$ & L. fermentum K.V9.3.2C & $62 \%$ \\
L. paracasei ES.D.88 & $84 \%$ & L. fermentum K.C6.3.1E & $53 \%$
\end{tabular}

*Results are expressed as a percentage of biofilm formation or elastolytic activity related to control ( $P$. aeruginosa PAO1 without lactobacilli). Only significantly active strains compared to control are displayed (LSD test, $p<0.05$ for biofilm formation and $p<0.001$ for elastolytic activity). erythromycin, clindamycin, quinupristin + dalfopristin, tetracycline, chloramphenicol).

\section{Lactobacilli growth and acidification properties in artificial saliva}

All the strains showed a good capacity to grow in artificial saliva with final concentrations after $48 \mathrm{~h}$ of incubation at $37^{\circ} \mathrm{C}$ comprised between $1.8 \times 10^{6}$ and $9.3 \times 10^{7} \mathrm{CFU} / \mathrm{ml}$ (Figure 2). Whatever the tested strain, no acidification occurred during the first $12 \mathrm{~h}(\mathrm{pH} 7.14 \pm 0.06)$ (Figure 3). The $\mathrm{pH}$ of artificial saliva however differed between tested strains after $24 \mathrm{~h}$ of incubation with a clear distinction between two groups. The first group (L. fermentum K.V9.3.2B, K.V9.3.2C, ES.A.2, and ES.F.115 and L. paracasei ES.D.88) was poorly acidifying with a decrease of $0.65 \pm$ $0.31 \mathrm{pH}$ units, whereas the second one (L. fermentum K. C6.3.1D, K.C6.3.1E and L. zeae Od.76) induced a much higher $\mathrm{pH}$ decrease with a diminution of $2.45 \pm 0.30$ units. However, after $36 \mathrm{~h}$ of incubation, the two facultative heterofermentative strains $L$. paracasei ES.D.88 and $L$. zeae Od.76 induced the highest $\mathrm{pH}$ decrease (minus $3.28 \pm 0.36 \mathrm{pH}$ units).

\section{Discussion}

$P$. aeruginosa is an opportunistic pathogen in patients with significant underlying diseases. It is one of the most common causes of hospital-acquired pneumonia, especially in mechanically ventilated patients, in whom it leads to a high mortality rate [2,17]. Moreover, chronic airway inflammation with recurrent $P$. aeruginosa infections is the major cause of morbidity and mortality in patients with cystic fibrosis [18]. High incidence, infection severity and increasing resistance characterizing $P$. aeruginosa infections highlight the need for new therapeutic options. In that context, different attempts have been made to use probiotic bacteria for fighting $P$. aeruginosa pulmonary infections [19]. Lactobacilli are nonpathogenic bacteria closely associated with the human 
Table 4 Species assignation of Lactobacillus isolates based on MALDI-TOF analyses, 16S RNA and rpoA genes sequencing

\begin{tabular}{|c|c|c|c|c|c|c|c|c|}
\hline \multirow[t]{2}{*}{ Strain } & \multicolumn{2}{|c|}{ Mass spectrometry } & \multicolumn{2}{|c|}{ 16S RNA gene sequencing } & \multicolumn{2}{|c|}{$r p o A$ gene sequencing } & \multicolumn{2}{|c|}{ API $50 \mathrm{CHL}$} \\
\hline & Identification & Score* & Identification & $\begin{array}{c}\text { NCBI accession number } \\
\text { (GenBank) }\end{array}$ & Identification & $\begin{array}{c}\text { NCBI accession number } \\
\text { (GenBank) }\end{array}$ & Identification & Score \\
\hline ES.A.2 & L. fermentum & 1.916 & L. fermentum & [KC762296] & L. fermentum & {$[$ KC861367] } & L. fermentum & $96.3 \%$ \\
\hline ES.D.88 & L. paracasei & 2.458 & L. casei group & [KC762297] & L. paracasei & [KC861369] & L. paracasei & $98.4 \%$ \\
\hline ES.F.115 & L. fermentum & 2.21 & L. fermentum & [KC762298] & L. fermentum & [KC861368] & L. fermentum & $98.5 \%$ \\
\hline Od.76*** & $\begin{array}{l}\text { L. zeae } \\
\text { L. casei }\end{array}$ & $\begin{array}{l}2.089 \\
2.043\end{array}$ & L. casei group & [KC762299] & L. zeae/casei & [KC861370] & $-{ }^{* *}$ & $-{ }^{* *}$ \\
\hline K.C6.3.1D & L. fermentum & 2.18 & L. fermentum & [KC762300] & L. fermentum & [KC861371] & L. fermentum & $99.7 \%$ \\
\hline K.C6.3.1E & L. fermentum & 2.109 & L. fermentum & [KC762301] & L. fermentum & [KC861372] & L. fermentum & $99.7 \%$ \\
\hline K.V9.3.2B & L. fermentum & 2.163 & L. fermentum & [KC762302] & L. fermentum & [KC861373] & L. fermentum & $99.7 \%$ \\
\hline K.V9.3.2C & L. fermentum & 2.223 & L. fermentum & [KC762303] & L. fermentum & [KC861374] & L. fermentum & $99.8 \%$ \\
\hline
\end{tabular}

${ }^{*}$ An isolate was considered well identified at the species level with a MALDI-TOF mass spectrometry score $\geq 1.9$. ${ }^{* * T h e}$ API $50 \mathrm{CHL}$ gallery is not designed to identify $L$. zeae. ${ }^{* * *}$ Od.76 was formally identified as a L. zeae by sequencing the pheS gene (Genbank accession number: KJ402364).

microbiota and commonly used as probiotics. Some of them are used because of their positive effects on the immune system, on the barrier effect of epithelia, whereas others are used for their capacity to fight pathogens colonisation either via competitive exclusion or antimicrobial molecules production. Probiotic effects are strain-specific, consequently they do not possess the same activity and they are not all recommended for the same health effects [20]. Specific selection criteria are then needed in order to find the right probiotic harbouring the appropriated activity (inhibition of pathogen for example) within a particular ecological niche. Some Lactobacillus spp. (L. rhamnosus
GG, L. plantarum 299, L. paracasei, L. casei, L. acidophilus), administered by oropharyngeal application or via orogastric or nasogastric tube, have already been tested, with different levels of success, in mechanically ventilated patients to fight $P$. aeruginosa pneumonia $[11,21]$. To our knowledge, none of them was specifically selected according to its capacity to inhibit $P$. aeruginosa, nor to survive in the oral cavity or in the oropharynx. The main goal of this work was then to test the capacity of lactobacilli isolated from oral cavities of healthy volunteers and from raw milk to inhibit the production of virulence factors by $P$. aeruginosa PAO1 in order to look for

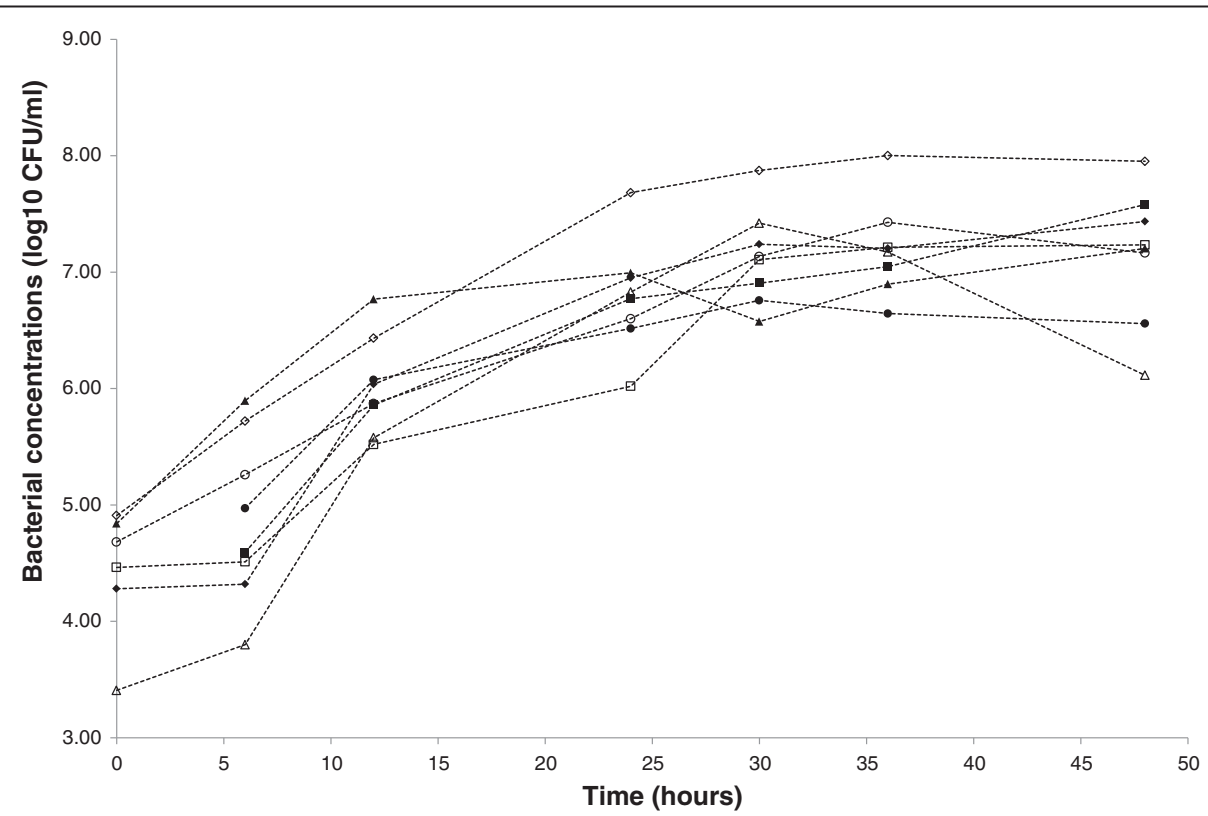

Figure 2 Growth of selected strains in artificial saliva. Bacterial concentrations ( $\log _{10}$ CFU/mL) of L. fermentum ES.A.2 ( $\triangle$ ), ES.F.115 (o),

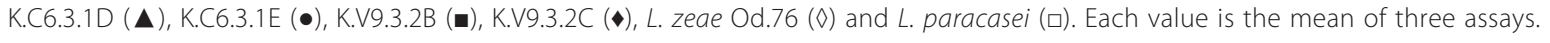




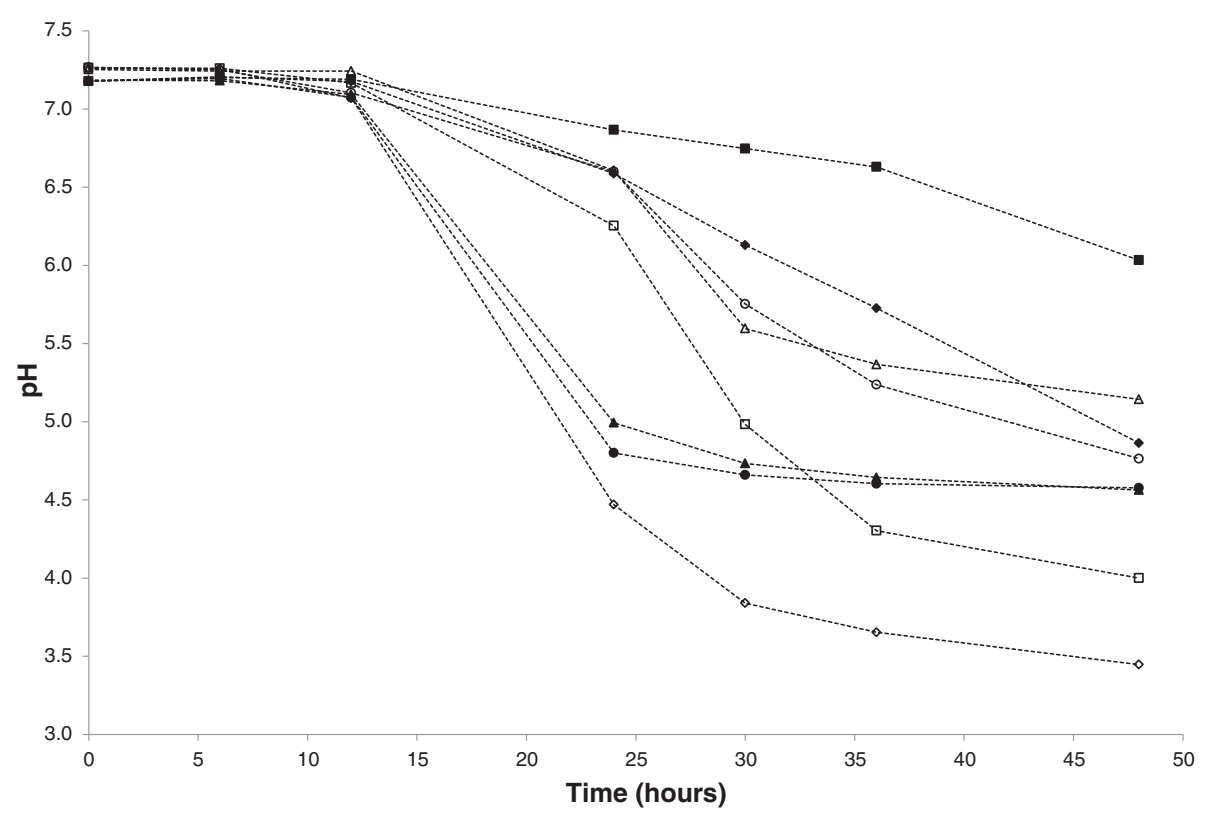

Figure 3 Acidification of the artificial saliva during the growth of selected strains. Acidifying properties of L. fermentum ES.A.2 ( $(\Delta)$, ES.F.115 (O),

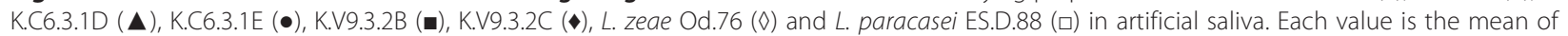
$\mathrm{pH}$ of three assays.

potential probiotic bacteria capable to prevent $P$. aeruginosa pneumonia.

In this study, 67 isolates belonging to 9 Lactobacillus species (L. reuteri, L. fermentum, L. vaginalis, L. rhamnosus, L. zeae, L. paracasei, L. salivarius and $L$. plantarum), with a prevalence of $L$. fermentum and $L$. paracasei, were recovered from the oral cavities of 23 healthy volunteers. The diversity of lactobacilli isolated from the oral cavity is generally high, and these 9 species are commonly encountered in healthy persons [22-27]. Since it has been suggested that oral lactobacilli may originate from the food [28], 20 Lactobacillus strains (L. fermentum, L. brevis and L. parabuchneri) isolated from raw milk and whom certain species have been previously described in the oral cavity were added to increase the pool of the tested strains. Although lactobacilli do not belong to the predominant oral microbiota, in which they account for less than $1 \%$ of the cultivable fraction, they are suspected to have a considerable effect on the homeostasis of this ecosystem [29].

Among the 87 tested isolates, the 10 most active ones against $P$. aeruginosa virulence factors were identified at the species level using a polyphasic approach combining phenotypic (MALDI-TOF, API $50 \mathrm{CHL}$ ) and molecular (16S rRNA and rpoA genes sequencing) methods, whereas typing of $L$. fermentum strains was performed using PFGE.

Finally 8 strains (six $L$. fermentum, one $L$. paracasei and one $L$. zeae) showed a significant inhibitory effect against $P$. aeruginosa PAO1 biofilm formation or elastolytic activity. With the exception of $L$. zeae and $L$. paracase $i$ that are facultative heterofermentative, all the active strains belonged to the L. fermentum species and were then obligate heterofermentative, producing both lactic and acetic acids from glucose. It has been shown that one of the major antibacterial effects of lactobacilli is mediated via lactic and acetic acids production [15]. Indeed, lactobacilli may produce high concentrations of lactic acid and acetic acid depending on their fermentative pathways and growth conditions. We have shown that $P$. aeruginosa $\mathrm{PAO} 1$ was sensitive to $\mathrm{pH}$ and acetic acid with a dose-dependent effect, growth inhibition increasing in parallel with an increase in acid concentration and $\mathrm{pH}$ decrease. At a pH of 4 or 5 , acetic acid completely inhibited the growth of $P$. aeruginosa at a concentration of $25 \mathrm{mM}$, whereas high concentrations $(\geq 50 \mathrm{mM})$ of acetic acid were necessary to partially inhibit $P$. aeruginosa growth at $\mathrm{pH}$ 6. For that reason, the inhibitory activities of lactobacilli toward $P$. aeruginosa PAO1 biofilm formation and elastolytic activity were not tested in MRS medium that contains a high glucose concentration $(20 \mathrm{~g} / \mathrm{L})$, but in BHI medium. Indeed, this medium contains a low concentration of glucose $(2 \mathrm{~g} / \mathrm{L})$ that limits the growth of Lactobacillus strains and prevents a strong acidification, allowing a better differentiation between the organic acids effects from other mechanisms of action.

Elastolytic activity and biofilm formation are two majors virulence factors observed in P. aeruginosa. Among the 8 strains $(6 L$. fermentum, one L. paracase $i$ and one L. zeae) significantly inhibiting elastase activity or biofilm formation, it is interesting to note that the four $L$. fermentum strains of milk origin (L. fermentum K.C6.3.1D, 
K.C6.3.1E, K.V9.3.2B and K.V9.3.2C) inhibited elastolytic activity only, whereas the ones originating from the oral cavity (L. fermentum ES.A2, ES.F.115) inhibited biofilm formation only. The two other active strains from the oral cavity, L. zeae Od.76 and L. paracasei ES.D.88 significantly inhibited elastase activity and biofilm formation respectively. Elastase has been shown to destroy respiratory epithelium tight junctions, increasing permeability disorders and interleukin-8 levels while decreasing host immune response [30,31]. We previously showed in a murine model of $P$. aeruginosa pneumonia, that elastolytic activity was positively correlated to acute lung injury [5]. It has been shown by Rumbaugh et al. that elastolytic activity and biofilm formation are under control of the quorum sensing molecules of $P$. aeruginosa [32]. Different mechanisms of action may then be hypothesized, active Lactobacillus strains inhibited the quorum sensing targets, either they secreted antagonistic analogues of acyl-homoserine lactone or they inhibited regulating lasR or lasI genes factors [33]. Moreover, the use of the BHI medium that induced a limited $\mathrm{pH}$ decrease, together with the low number of active strains, suggested that other mechanisms of action than organic acids production were implicated. It has been shown that surface properties, such as cell charge and hydrophobicity, implicated in the non-specific adhesive capacity of bacteria differ among Lactobacillus strains isolated from the oral cavity of healthy volunteers, with several strains (including strains of $L$. fermentum and $L$. paracasei) showing very high adhesive properties [23]. Such a difference in surface properties between lactobacilli strains with a prevalence of high adhesive properties in lactobacilli strains isolated from the mouth might be implicated in their higher capacity to prevent biofilm formation as compared to dairy lactobacilli. Indeed surface properties are involved in adhesion properties to plastic and/or in the co-aggregation with $P$. aeruginosa that could be implicated in decreasing biofilm formation. However, further studies are needed to elucidate the antagonistic mechanism of action between described lactobacilli strains and $P$. aeruginosa.

Antagonistic activities of probiotic bacteria require a certain capacity to survive and/or to grow in the targeted ecosystem. All active strains showed a good capacity to grow in artificial saliva, suggesting that they may survive in the oral environment. However, it has been suggested that some probiotics may be implicated in the development of dental caries [34]. The use of poorly acidifying strains such as L. fermentum K.V9.3.2B and K. V9.3.2C inhibiting elastolytic activity and L. fermentum ES.F.115 and ES.A.2 inhibiting biofilm formation may then be encouraged in their use as probiotics to fight $P$. aeruginosa pulmonary infection compared to the more acidifying ones. However, knowing that acid production strongly inhibits $P$. aeruginosa growth, the use of more acidifying strains may be also investigated and subjected to an appropriate follow-up of dental health during probiotic application. Another theoretical concern regarding the safety of probiotics is the transfer of antibiotic resistance genes toward the oral and gastrointestinal microbiota. In our study, as expected for lactobacilli that are intrinsically resistant to vancomycin, all the strains were resistant to vancomycin [35]. No other resistance towards the recommended antibiotics was detected. On the other hand the toxic effect of putative probiotic on the epithelial cells from the oropharynx and respiratory tract will have to be investigated.

\section{Conclusions}

The pathogenesis of ventilator acquired pneumonia requires micro-aspiration of oropharynx microbiota into the lower airway. The natural oropharynx microbiota of patients is modified by exogenous bacteria from the hands of the health care workers for example or by endogenous bacteria such as the intestinal microbiota by retrograde contamination. In our study, we screened 87 lactobacilli isolates from the oral cavity of healthy people and from milk with the aim to prevent $P$. aeruginosa from colonising the oropharynx environment. Eight strains, devoid of acquired antibiotic resistance were able to grow in artificial saliva and to decrease two virulence factors (elastolytic activity and biofilm formation) of $P$. aeruginosa in vitro. The next step will be to check if these strains induce a significant protective effect in an in vivo model of $P$. aeruginosa pneumonia.

\section{Methods}

\section{Ethics}

Lactobacilli from swab samples belong to the biological collection DC-2008-214 of Brest University Hospital. This biological collection was approved by Ministry of Higher Education and Research. Our study was approved by our Medical Hospital University ethics committee («Comité de Protection des Personnes Ouest VI»). A written informed consent was obtained from all healthy adults volunteers participating in the study for publication of this case report. A copy of the written consent is available for review by the Editor-in-Chief of this journal. There were no under age children ( $<18$ years-old) included in our study.

\section{Bacterial strains and culture conditions}

$P$. aeruginosa PAO1, initially isolated from an infected wound [36], was chosen as reference strain for the activity tests. It was routinely cultivated overnight at $37^{\circ} \mathrm{C}$ in Luria Bertani (LB), or in Brain Heart Infusion broth (BHI) prior to the activity experiments. A total of 87 Lactobacillus isolates were tested for their capacity to inhibit $P$. aeruginosa PAO1 biofilm formation and 
elastolytic activity. Twenty of them belonged to a collection of Lactobacillus spp. (15 L. fermentum, 1 L. brevis and 4 L. parabuchneri) previously isolated from raw milks in our laboratory [13], and 67 were isolated from the mouth of healthy volunteers during this work (see below). Lactobacillus fermentum ATCC 9338 was obtained from AES Chemunex (Bruz, France), whereas Lactobacillus casei LMG 6904 and Lactobacillus paracasei LMG 13087 were obtained from the BCCM/LMG Bacteria Collection (http://bccm.belspo.be/about/lmg.php). They were used as reference strains for lactobacilli identification. Lactobacilli were cultured in de Mann, Rogosa and Sharpe (MRS) broth at $37^{\circ} \mathrm{C}$. All strains were maintained as $33 \%$ glycerol stock at $-80^{\circ} \mathrm{C}$. All bacterial growth media were purchased from AES Chemunex, Bruz, France if not otherwise stated.

\section{Isolation and identification of oral lactobacilli}

Twenty-three healthy adult volunteers were asked to rub the inside of their mouth with sterile swabs, which were then used to inoculate LAMVAB, a selective agar medium for the isolation of lactobacilli [37]. After $48 \mathrm{~h}$ of incubation at $37^{\circ} \mathrm{C}$, single colonies were picked-up and transplanted at least thrice on MRS agar for isolation. Isolates were examined by phase-contrast microscopy, Gram stained and tested for the absence of catalase. They were then assigned to the Lactobacillus genus by MALDI-TOF mass spectrometry analyses with protein extraction [13]. Once several isolates were described as effective against $P$. aeruginosa PAO1, they were formally identified by sequencing their $16 S$ rRNA, rpoA and pheS genes, using respectively U1/RU2, rpoA-21-F/rpoA-23-R and pheS-21-F/pheS-22-R primers on their total DNA [38,39]. Part of the $16 S r R N A$ gene was amplified with an initial denaturation step performed for $15 \mathrm{~min}$ at $95^{\circ} \mathrm{C}$, followed by 30 amplifications cycles consisting of $1 \mathrm{~min}$ at $95^{\circ} \mathrm{C}$ for denaturation, $30 \mathrm{~s}$ at $64^{\circ} \mathrm{C}$ for primer annealing, $1 \mathrm{~min}$ at $72^{\circ} \mathrm{C}$ for extension, and one final extension of $5 \mathrm{~min}$ at $72^{\circ} \mathrm{C}$. For the rpo $A$ and pheS genes, an initial denaturation step was performed for $5 \mathrm{~min}$ at $95^{\circ} \mathrm{C}$, followed by 3 amplification cycles of $1 \mathrm{~min}$ at $95^{\circ} \mathrm{C}, 2 \mathrm{~min} 15 \mathrm{~s}$ at $46^{\circ} \mathrm{C}, 1 \mathrm{~min} 15 \mathrm{~s}$ at $72^{\circ} \mathrm{C}$, and 30 cycles of $35 \mathrm{~s}$ at $95^{\circ} \mathrm{C}$, $1 \mathrm{~min} 15 \mathrm{~s}$ at $46^{\circ} \mathrm{C}, 1 \mathrm{~min} 15 \mathrm{~s}$ at $72^{\circ} \mathrm{C}$ and a final extension step of $7 \mathrm{~min}$ at $72^{\circ} \mathrm{C}$. In few cases, an annealing temperature of $42^{\circ} \mathrm{C}$ was used for the amplification of rpoA or pheS. PCR amplicons were sequenced either at the molecular genetic department sequencing platform in the "CHRU de Brest" (INSERM-U1078) or at the Biogenouest platform (Roscoff, France). Sequences were then assembled using DNA Baser 3.5.3, and aligned using the MAFFT tool (http://mafft.cbrc.jp/alignment/server/) (December 2012) for species assignment. Phylogenetic analyses were conducted by the neighbour-joining method using MEGA 5.05/5.10 software [40]. Bacterial sequences for the sequenced strains have been deposited in GenBank and accession numbers are available in Table 4. Biochemical profiles were analysed by using API $50 \mathrm{CHL}$ test kit (Biomérieux, Marcy l'Étoile, France). Isolates belonging to the L. fermentum species were then typed with PFGE analyses as described by Delavenne et al. [41].

\section{Effects of $\mathrm{pH}$ and organic acids on $P$. aeruginosa PAO1 growth}

An overnight culture of $P$. aeruginosa PAO1 was harvested by centrifugation, washed, and suspended in the same volume of saline solution $(\mathrm{NaCl} 0.9 \%)$. One hundred microliters of this suspension was then used to inoculate $10 \mathrm{ml}$ of LB supplemented with acetic acid (50 or $100 \mathrm{mM})$ or lactic acid $(12.5,25,50$ or $100 \mathrm{mM})$ at different $\mathrm{pH}(4,5,6$ and 7$)$. The growth of $P$. aeruginosa PAO1 was monitored by measuring the $\mathrm{OD}_{600 \mathrm{~nm}}$ after $22 \mathrm{~h}$ of incubation at $37^{\circ} \mathrm{C}$. Negative controls consisted in culturing $P$. aeruginosa PAO1 with no acetic or lactic acids but with hydrochloric acid $(\mathrm{HCl})$ in order to reach the desired $\mathrm{pH}$. All the tests were performed in triplicate.

\section{Inhibition of $P$. aeruginosa biofilm formation}

The capacity of Lactobacillus isolates to inhibit biofilm formation by $P$. aeruginosa PAO1 was tested with a colorimetric method adapted from Merrit and Valdez $[42,43]$. P. aeruginosa PAO1 and lactobacilli were cultivated overnight at $37^{\circ} \mathrm{C}$ separately in $\mathrm{BHI}$ broths. After incubation, the two bacterial suspensions were washed with a saline solution, diluted in BHI ( $\mathrm{pH}$ 7.4), and mixed in order to obtain a final suspension containing $5 \times 10^{7} \mathrm{CFU} / \mathrm{ml}$ of $P$. aeruginosa and $5 \times 10^{7} \mathrm{CFU} / \mathrm{ml}$ of the tested Lactobacillus isolate. One hundred millilitres of this bacterial suspension was deposited per well in 96-well flat-bottomed microplates (Corning Incorporated, Corning, USA), that were incubated at $37^{\circ} \mathrm{C}$. After $7 \mathrm{~h}$, the wells were washed twice with a saline solution $(\mathrm{NaCl} 0.9 \%)$, and $100 \mu \mathrm{l}$ of crystal violet (0.25\%) were added in each well for biofilm colouring. After $10 \mathrm{~min}$, wells were washed twice again with the saline solution and the remaining crystal violet was released by addition of $100 \mu \mathrm{l}$ of acetic acid (33\%). Finally, the acetic acid solution containing the released crystal violet was transferred in a new microplate and the $\mathrm{OD}_{595 \mathrm{~nm}}$ was measured using a spectrophotometer (Multiskan FC Microplate Photometer, Thermo Scientific, Waltham USA). The positive control was the amount of biofilm formed with a pure culture of $P$. aeruginosa PAO1, whereas the negative control was sterile BHI. Three series of four wells were performed. In parallel, the viability of $P$. aeruginosa on co-cultures with lactobacilli during 7 hours was evaluated by plate counts. Acidification was quantified through $\mathrm{pH}$ measurement. 


\section{Inhibition of elastolytic activity of $P$. aeruginosa}

The capacity of Lactobacillus isolates to inhibit the elastolytic activity of $P$. aeruginosa PAO1 was tested with a colorimetric method adapted from Rust et al. [44]. Aliquots $(2 \mathrm{ml})$ of bacterial suspensions ( $P$. aeruginosa PAO1 at $5 \times 10^{7} \mathrm{CFU} / \mathrm{ml}$ and Lactobacillus isolate at $5 \times 10^{7} \mathrm{CFU} /$ $\mathrm{ml}$ ) used for the biofilm experiment were also used in the elastase assay. They were incubated at $37^{\circ} \mathrm{C}$ for $22 \mathrm{~h}$, centrifuged at $2000 \times g$ for $5 \mathrm{~min}$, and one $\mathrm{ml}$ of a solution of elastin Congo-red $(20 \mathrm{mg} / \mathrm{ml}$; Sigma-Aldrich, St Louis, USA) in a $10 \mathrm{mM}$ sodium phosphate buffer ( $\mathrm{pH} 7.0$ ) was added to the supernatant that was incubated at $37^{\circ} \mathrm{C}$ for $18 \mathrm{~h}$. The insoluble elastin Congo-red was pelleted at 2 $000 \times g$ for $20 \mathrm{~min}$ and the absorbance of the Congo-red soluble fraction released by elastase, was measured at $450 \mathrm{~nm}$ with a spectrophotometer (Multiskan* FC Microplate Photometer, Thermo Scientific). The positive control was the elastolytic activity in a pure culture of $P$. aeruginosa PAO1 and the negative control was sterile BHI. Three series of experiments were performed. The viability of $P$. aeruginosa on co-cultures with lactobacilli during 22 hours was evaluated by plate counts. Acidification was quantified through $\mathrm{pH}$ measurement.

\section{Sensitivity of lactobacilli to antibiotics}

Lactobacillus isolates were tested for their susceptibility to a panel of 10 antibiotics (ampicillin, vancomycin, gentamicin, kanamycin, streptomycin, erythromycin, clindamycin, quinupristin + dalfopristin, tetracycline, chloramphenicol). The screening was performed with Etests (Etest ${ }^{\circ}$, Biomérieux, Marcy l'Étoile, France) following instructions as indicated by the producer. Lactobacilli were cultivated on MRS agar during $48 \mathrm{~h}$, colonies were picked-up and suspended in $5 \mathrm{ml}$ of a saline solution $(0.9 \% \mathrm{NaCl})$ to obtain a McFarland standard OD of 0.5, and five drops of this suspension were added to $10 \mathrm{ml}$ of a new saline solution. The suspension was finally spread on blood agar plates (AES Chemunex, Bruz, France), the excess liquid was discarded, and the Etests stripes were applied to the dried plates. The minimal inhibition concentrations (MIC), expressed in $\mathrm{mg} / \mathrm{L}$, were read on the Etest stripes after $48 \mathrm{~h}$ of incubation at $37^{\circ} \mathrm{C}$. Results were interpreted according to the cut-off levels proposed for Enterococcus spp. by the Committee of the antibiogram of the French society for microbiology $[41,45]$.

\section{Lactobacilli growth and acidification properties in artificial saliva}

The growth in artificial saliva of several Lactobacillus strains (L. zeae Od.76, L. paracasei ES.D.88 and L. fermentum ES.A.2, ES.F.115, K.C6.3.1D and K.V9.3.2C) was regularly monitored by determining the $\mathrm{pH}$ and plate counts on MRS agar during $48 \mathrm{~h}$ of incubation at $37^{\circ} \mathrm{C}$. Artificial saliva composition was previously described by
Roger et al. [46]. Initial concentrations of tested isolates were set around $5 \log _{10} \mathrm{CFU} / \mathrm{ml}$. All experiments were carried out in triplicate.

\section{Statistics}

Statistical analyses were performed using the Microsoft Excel 2010 (Microsoft Corporation, Redmond, USA) and SAS 9.3 (SAS Institute Inc., Cary, USA) software products. In order to test the significance of the elastolytic activity and biofilm formation assays, the correlation between the three series of experiments was first assessed by the Student's inverse test $(p<0.05)$. Once the correlation between the assays was established, significant differences within each assay were carried out with a one-way analysis of variance (ANOVA), and the least significant difference (LSD) test was used to detect antagonistic activities of lactobacilli strains against Pseudomonas aeruginosa. Statistical significance were set at a $p=0.001$ for elastolytic activity and $p=0.05$ for biofilm formation. Organic acids and $\mathrm{pH}$ effects on $P$. aeruginosa $\mathrm{PAO} 1$ growth were studied by performing covariance analyses (ANCOVA) on optical densities.

\section{Additional file}

Additional file 1: Fermentation patterns of active strains. This table presents the whole results obtained with the API $50 \mathrm{CHL}$ gallery for the 8 tested strains.

\section{Competing interests}

The authors declare they have no competing interests.

\section{Authors' contributions}

RLB and GLB lead the study and drafted the manuscript. YA performed the most part of the assays and drafted the manuscript. GB performed the statistics. All authors read and approved the final manuscript.

\section{Acknowledgements}

The authors thank Stéphanie Gouriou, Stella Debaets, Franck Déniel and Jérôme Mounier for their implication in the sequencing of Lactobacillus spp. strains; Emmanuel Nowak for his work on statistics (INSERM CIC 1412, Brest, France); Danielle Arzur for help in performing biofilm and elastolytic assays; Michelle Virmaux for support in PFGE analysis, and Geneviève Héry-Arnaud who provided an access to the mass spectrometry platform, and Zarrin Alavi Menut for her pertinent advice for submitting this manuscript (INSERM CIC 1412, Brest, France). This work was supported by a PhD fellowship from the French Ministry of Higher Education and Research.

\section{Author details}

'Université de Brest, EA 3882-Laboratoire Universitaire de Biodiversité et d'Écologie Microbienne (LUBEM), Faculté de Médecine, 22 avenue Camille Desmoulins, 29200 Brest, France. ²Département de Médecine Interne et Pneumologie, CHRU La Cavale-Blanche, 29200 Brest, France. ${ }^{3}$ Université de Brest, EA 3882-Laboratoire Universitaire de Biodiversité et d'Écologie Microbienne (LUBEM), Parvis Blaise Pascal, Technopôle Brest-Iroise, 29280 Plouzané, France. ${ }^{4}$ Université de Brest, CNRS, IFREMER, UMR 6197-Laboratoire de Microbiologie des Environnement Extrêmes (LMEE), Institut Universitaire Européen de la Mer, Place Nicolas Copernic, Technopôle Brest-Iroise, 29280 Plouzané, France.

Received: 13 February 2014 Accepted: 22 April 2014 Published: 27 April 2014 


\section{References}

1. Gaynes R, Edwards JR: Overview of nosocomial infections caused by Gram-negative bacilli. Clin Infect Dis 2005, 41:848-854

2. Fujitani S, Sun H-Y, Yu VL, Weingarten JA: Pneumonia due to Pseudomonas aeruginosa. Part I: epidemiology, clinical diagnosis, and source. Chest 2011, 139:909-919.

3. Rajan S, Saiman L: Pulmonary infections in patients with cystic fibrosis. Semin Respir Infect 2002, 17:47-56.

4. Sadikot RT, Blackwell TS, Christman JW, Prince AS: Pathogen-host interactions in Pseudomonas aeruginosa pneumonia. Am J Respir Crit Care Med 2005, 171:1209-1223.

5. Le Berre R, Nguyen S, Nowak E, Kipnis E, Pierre M, Quenee L, Ader F, Lance S, Courcol R, Guery BP, Faure K: Relative contribution of three main virulence factors in Pseudomonas aeruginosa pneumonia. Crit Care Med 2011, 39:2113-2120.

6. Singh PK, Schaefer AL, Parsek MR, Moninger TO, Welsh MJ, Greenberg EP. Quorum-sensing signals indicate that cystic fibrosis lungs are infected with bacterial biofilms. Nature 2000, 407:762-764.

7. Guinane CM, Cotter PD: Role of gut microbiota in health and chronic gastrointestinal disease: understanding a hidden metabolic organ. Ther Adv Gastroenterol 2013, 6:295-308.

8. Wang Z, Yang Y: Upper gastrointestinal microbiota and digestive diseases. World J Gastroenterol 2013, 19:1541-1550.

9. Round $J$, Mazmanian SK: The gut microbiota shapes intestinal immune responses during health and disease. Nat Rev Immunol 2009, 9:313-323.

10. Meurman JH: Probiotics: do they have a role in oral medicine and dentistry? Eur J Oral Sci 2005, 113:188-196.

11. Siempos II, Ntaidou TK, Falagas ME: Impact of the administration of probiotics on the incidence of ventilator-associated pneumonia: a meta-analysis of randomized controlled trials. Crit Care Med 2010, 38:954-962.

12. Hao Q, Lu Z, Dong BR, Huang CQ, Wu T: Probiotics for preventing acute upper respiratory tract infections. Cochrane Database Syst Rev 2011,CD006895.

13. Delavenne E, Mounier J, Déniel F, Barbier G, Le Blay G: Biodiversity of antifungal lactic acid bacteria isolated from raw milk samples from cow, ewe and goat over one-year period. Int J Food Microbiol 2012, 155:185-190.

14. Felis GE, Dellaglio F: Taxonomy of lactobacilli and bifidobacteria. Curr Issues Intest Microbiol 2007, 8:44-61.

15. Alakomi H-LL, Skyttä E, Saarela M, Mattila-Sandholm T, Latva-Kala K, Helander IM: Lactic acid permeabilizes gram-negative bacteria by disrupting the outer membrane. Appl Environ Microbiol 2000, 66:2001-2005.

16. Phillips I, Lobo AZ, Fernandes R, Gundara NS: Acetic acid in the treatment of superficial wounds infected by Pseudomonas aeruginosa. Lancet 1968 $1: 11-14$

17. Crouch Brewer S, Wunderink RG, Jones CB: Leeper K V: Ventilator-associated pneumonia due to Pseudomonas aeruginosa. Chest 1996, 109:1019-1029.

18. Khan TZ, Wagener JS, Bost T, Martinez J, Accurso FJ, Riches DW: Early pulmonary inflammation in infants with cystic fibrosis. Am J Respir Crit Care Med 1995, 151:1075-1082

19. Alexandre Y, Le Blay G, Boisramé-Gastrin S, Le Gall F, Héry-Arnaud G, Gouriou S, Vallet S, Le Berre R: Probiotics: a new way to fight bacterial pulmonary infections? Médecine Mal Infect 2013, 44:9-17.

20. Petrof EO: Probiotics and gastrointestinal disease: clinical evidence and basic science. Antiinflamm Antiallergy Agents Med Chem 2009, 8:260-269.

21. Barraud D, Blard C, Hein F, Marçon O, Cravoisy A, Nace L, Alla F, Bollaert P-E, Gibot S: Probiotics in the critically ill patient: a double blind, randomized, placebo-controlled trial. Intensive Care Med 2010, 36:1540-1547.

22. Dewhirst FE, Chen T, Izard J, Paster BJ, Tanner ACR, Yu W-H, Lakshmanan A, Wade WG: The human oral microbiome. J Bacteriol 2010, 192:5002-5017.

23. Colloca M, Ahumada M, López M, Nader-Macías M: Surface properties of lactobacilli isolated from healthy subjects. Oral Dis 2008, 6:227-233.

24. Teanpaisan R, Dahlén G: Use of polymerase chain reaction techniques and sodium dodecyl sulfate-polyacrylamide gel electrophoresis for differentiation of oral Lactobacillus species. Oral Microbiol Immunol 2006, 21:79-83.

25. Ahrné S, Nobaek S, Jeppsson B, Adlerberth I, Wold A, Molin G: The normal Lactobacillus flora of healthy human rectal and oral mucosa. J Appl Microbiol 1998, 85:88-94.

26. Dal Bello F, Hertel C: Oral cavity as natural reservoir for intestinal lactobacilli. Syst Appl Microbiol 2006, 29:69-76.

27. Maukonen J, Mättö J, Suihko M-L, Saarela M: Intra-individual diversity and similarity of salivary and faecal microbiota. J Med Microbio/ 2008 57:1560-1568.
28. Caufield PW, Li Y, Dasanayake A, Saxena D: Diversity of lactobacilli in the oral cavities of young women with dental caries. Caries Res 2007, 41:2-8

29. Meurman JH, Stamatova I: Probiotics: contributions to oral health. Oral Dis 2007, 13:443-451.

30. Kon Y, Tsukada H, Hasegawa T, Igarashi K, Wada K, Suzuki E, Arakawa M, Gejyo F: The role of Pseudomonas aeruginosa elastase as a potent inflammatory factor in a rat air pouch inflammation model. FEMS Immunol Med Microbiol 1999, 25:313-321.

31. Azghani $A O$, Bedinghaus $T$, Klein R: Detection of elastase from Pseudomonas aeruginosa in sputum and its potential role in epithelial cell permeability. Lung 2000, 178:181-189.

32. Rumbaugh KP, Griswold JA, Hamood AN: The role of quorum sensing in the in vivo virulence of Pseudomonas aeruginosa. Microbes Infect 2000, 2:1721-1731

33. Smith RS, Iglewski BH: Pseudomonas aeruginosa quorum sensing as a potential antimicrobial target. J Clin Invest 2003, 112:1460-1465.

34. Montalto M, Vastola M, Marigo L, Covino M, Graziosetto R, Curigliano V, Santoro L, Cuoco L, Manna R, Gasbarrini G: Probiotic treatment increases salivary counts of lactobacilli: a double-blind, randomized, controlled study. Digestion 2004, 69:53-56

35. Danielsen $M$, Wind $A$ : Susceptibility of Lactobacillus spp to antimicrobial agents. Int J Food Microbiol 2003, 82:1-11.

36. Holloway BW: Genetic recombination in Pseudomonas aeruginosa. J Gen Microbiol 1955, 13:572-581.

37. Hartemink R, Domenech V, Rombouts F: LAMVAB-A new selective medium for the isolation of lactobacilli from faeces. J Microbiol Methods 1997, 29:77-84.

38. Héry-Arnaud G, Rouzic N, Doloy A, Le Lay G, Garré M, Payan C, Poyart C Streptococcus australis meningitis. J Med Microbiol 2011, 60:1701-1704.

39. Naser SM, Thompson FL, Hoste B, Gevers D, Dawyndt P, Vancanneyt M, Swings J: Application of multilocus sequence analysis (MLSA) for rapid identification of Enterococcus species based on rpoA and pheS genes. Microbiology 2005, 151:2141-2150.

40. Tamura K, Peterson D, Peterson N, Stecher G, Nei M, Kumar S: MEGA5: molecular evolutionary genetics analysis using maximum likelihood, evolutionary distance, and maximum parsimony methods. Mol Biol Evol 2011, 28:2731-2739.

41. Delavenne E, Ismail R, Pawtowski A, Mounier J, Barbier G, Le Blay G: Assessment of lactobacilli strains as yogurt bioprotective cultures. Food Control 2013, 30:206-213.

42. Merritt JH, Kadouri DE, OToole GA, Toole GAO: Growing and analyzing static biofilms. Curr Protoc Microbiol 2005, 00:1B.1.1-1B.1.17.

43. Valdéz JC, Peral MC, Rachid M, Santana M, Perdigón G: Interference of Lactobacillus plantarum with Pseudomonas aeruginosa in vitro and in infected burns: the potential use of probiotics in wound treatment. Clin Microbiol Infect 2005, 11:472-479.

44. Rust L, Messing CR, Iglewski BH: Elastase assays. Methods Enzymol 1994, 235:554-562.

45. Soussy CJ, Bonnet R, Caron F, Cavallo JD, Chardon H, Chidiac C, Courvalin P, Drugeon $H$, Dubreuil $L$, Jarlier $V$, Jehl F, Lambert T, Leclerca R, NicolasChanoine MH, Plesiat P, Ploy MC, Quentin C, Varon E, Weber P: Comité de l'antibiogramme de la société française de microbiologie. Recommandations 2012, 2012:59p

46. Roger P, Delettre J, Bouix M, Béal C: Characterization of Streptococcus salivarius growth and maintenance in artificial saliva. J App/ Microbiol 2011, 111:631-641.

\section{doi:10.1186/1471-2180-14-107}

Cite this article as: Alexandre et al: Screening of Lactobacillus spp. for the prevention of Pseudomonas aeruginosa pulmonary infections. BMC Microbiology 2014 14:107. 